\title{
Comparative Study of Different Therapies in Glaucoma
}

\author{
Yan Liu' ${ }^{1}$, Jie Su${ }^{1}$ and Xiao Zhang ${ }^{2}$ \\ ${ }^{1}$ Affiliated Hospital of North China University of Science and Technology, 063000 \\ ${ }^{2}$ North China University of Science and Technology, 063000
}

Keywords: Trabeculectomy; Glaucoma; Mitomycin C

\begin{abstract}
Objective: To compare the efficacy of trabeculectomy combined with mitomycin C in the treatment of glaucoma and trabeculectomy alone. Methods: This study selected our hospital from March 2017 to December 2017 to come to my hospital ophthalmic treatment of 200 cases of glaucoma patients for the study of the study, and the patients were randomly divided into two groups, one for the control group, and the other observation group, in which the control group had 100 cases, the observation group had 100 cases, the control group received the standard trabecular surgery based on the suprachoroidal conjunctival flap, and the observation group was given small Beam resection combined with mitomycin $C$ wet compress were used to compare the curative effect of the two groups. Results: The proportion of patients with complete success in the observation group was significantly higher than that of the control group. The total success rate of the observation group was significantly higher than that of the control group. The postoperative satisfaction of the observation group was significantly higher than that of the control group. Conclusion: The efficacy of trabeculectomy combined with mitomycin $\mathrm{C}$ in the treatment of glaucoma is better than trabeculectomy combined with mitomycin $\mathrm{C}$ in the treatment of glaucoma.
\end{abstract}

\section{Introduction}

Glaucoma is a group of eye diseases which result in damage to the optic nerve and vision loss. The main risk factors for glaucoma include increased intraocular pressure and ischemia of optic nerve ${ }^{[1]}$. Beside, optic nerve damage and pressure damage may also associated with the occurrence and development of glaucoma. Obstruction of any part of the aqueous humor circulation pathway may cause elevated intraocular pressure, and finally lead to pathological changes. Conversely, optic nerve damage may occur with normal pressure, known as normal-tension glaucoma. It is one of the three major blindness diseases that cause blindness in human beings.

After 45 years of age, the total incidence rates were $1 \%$ to $2 \%$. According to the etiology, angle and intraocular pressure, it can be divided into three categories: open-angle glaucoma, closed-angle glaucoma and normal-tension glaucoma ${ }^{[2]}$. Congenital glaucoma is due to abnormal embryonic development---the variability of congenital corneal structure. Glaucoma is one of the main causes of blindness in Chinese patients ${ }^{[3]}$.

A lot of experience in clinical treatment has shown that vision loss from glaucoma may be permanent, so the treatment of glaucoma in patients should be given adequate attention. The current clinical treatment of glaucoma still advocates that physical examination likes screening via regular eye check-ups, will help timely diagnosis and give the patient early regular clinical treatment. If a patient who suffers from glaucoma can be found early and accessed reasonable clinical treatment timely, the patient's vision recovery effect may be prolonged. Therefore, glaucoma treatment advocates early treatment.

The modern goals of glaucoma management are to decrease eye pressure, glaucomatous damage and nerve damage, and preserve visual field. The most common conventional surgery performed for glaucoma is the trabeculectomy. However, causes such as obstruction of the filter passage can often lead to the failure of the operation, which affects the visual function and life quality of the patients ${ }^{[4]}$.

In recent years, the application of mitomycin $C$ in the treatment of glaucoma has been attached 
more attention. Mitomycin C is a mitomycin that is used as a chemotherapeutic agent by virtue of its antitumour activity. Mitomycin $\mathrm{C}$ is a antimetabolite, which can decompose cell DNA, block DNA replication and inhibit tumor cell division. It is a kind of antitumor antibiotic isolated from Streptomyces, which can inhibit the replication of proliferative DNA by destroying the structure and function of it. It is cytotoxic to cells and also works on quiescent cell. In eye surgery, mitomycin C $0.02 \%$ is applied topically to prevent scarring and decrease the production of fibroblasts and scar tissue during glaucoma filtering surgery ${ }^{[6]}$. Studies have shown that mitomycin $\mathrm{C}$ can effectively inhibit the differentiation and proliferation of desmocyte. Therefore, mitomycin $\mathrm{C}$ also has a significant effect in the treatment of glaucoma ${ }^{[7]}$.

This study selected 200 glaucoma patients from March 2017 to December 2017 in our hospital to evaluate the efficacy of trabeculectomy combined with mitomycin $C$ in the treatment of glaucoma.

\section{Materials and Methods}

\subsection{General materials}

This study selected 200 cases of glaucoma patients treated with ophthalmology from March 2017 to December 2017 in our hospital. the patients were randomly divided into two groups, one for the control group, and the other was observation group, in which the control group had 100 cases(males 57cases, females 43cases,averge age 67 \pm 2.5 ), the observation group had 100 cases(males 55cases, females 45cases, averge age 68 \pm 2.3 ). There was no significant difference in the patients' sex, age and patient's disease types.

\subsection{Research methods}

The control group received standard trabeculectomy based on the conjunctival flap above the fornix. The observation group, cotton piece were soaked in the concentration of $0.26 \sim 0.68 \mathrm{mg} / \mathrm{m}$ $\mathrm{L}$ mitomycin $\mathrm{C}$ solution. this cotton piece were applied in the sclera flap and the surrounding for 2 $\sim 5 \mathrm{~min}$, for refractory glaucoma with $0.4 \mathrm{~g} / \mathrm{L}, 15 \mathrm{~min}$, for primary glaucoma with $0.2 \mathrm{~g} / \mathrm{L}, 13$ min. then, remove the cotton, the operation area rinsed with saline $200 \mathrm{ml}$, the next dealing with the control group in the same way. In general one week after surgery patient can be discharge, check once a week, once a month, and followed up for 1 year. In addition, it is important to inform patients that mood swings can significantly affect the intraocular pressure fluctuations. Emotional ups and down the intraocular pressure will increase, the pressure of the eye to increase will aggravate glaucoma, so glaucoma patients should maintain a pleasant mood, optimistic and cheerful. Maintain a good mental state. Life must be regular. For example, don't smoke and drink, go to bed early and get up early. Don't eat spicy food. Don't overeat and other habits. A healthy lifestyle can reduce the incidence of glaucoma and help control the condition of glaucoma patients. In addition, it is suggested that you can drink two cups of "9 wei irises tea", accelerate blood circulation, prevent eye blood stasis, enhance the ability of eyes to resist high intraocular pressure, and persist in drinking to promote the improvement of glaucoma.

\subsection{The criteria of curative effect}

Evaluation of the curative effect of postoperative patients, the criteria is: intraocular pressure reaches 6-21 MMHG, patients who don't need anti-glaucoma medications after surgery, can be judged as completely successful operation, intraocular pressure reaches 6-21 MMHG, patients requiring topical anti-glaucoma medications after surgery, or patients with postoperative bindness, can be judged as the partial success of operation, postoperative intraocular pressure to achieve $>21$ MMHG, can also be judged as the partial success of operation. For patients who require further surgery to to treat glaucoma after operation, or who have long-term low intraocular pressure $(<6$ MMHG), or severe postoperative complications, or who have had loss of vision, can be judged as operation failure. 


\subsection{Statistical methods}

The statistical method was SPSS11.0 statistical software, counting data was compared with $\mathrm{X}^{2}$ test, and the measurement data was compared with $t$ test. $\mathrm{P}$ values $<0.05$ were considered to be Significant differences.

\section{Results}

Comparison of the postoperative curative effect between the two groups, in the observation group, the proportion of postoperative patients with complete success was significantly higher than the proportion of postoperative patients in the control group, and the total success rate of patients in the observation group was significantly higher than that in the control group.

Table 1 Comparison of the postoperative curative effect between the two groups

\begin{tabular}{|c|c|c|c|c|c|}
\hline group & $\mathrm{n}$ & $\begin{array}{l}\text { complete } \\
\text { succes }\end{array}$ & $\begin{array}{c}\text { partial } \\
\text { success }\end{array}$ & failure & total success \\
\hline observation group & 100 & 84 & 13 & 3 & 97 \\
\hline $\begin{array}{c}\text { Postoperative } 1 \\
\text { week }\end{array}$ & 100 & 87 & 7 & 6 & 94 \\
\hline $\begin{array}{l}\text { Postoperative } 3 \\
\text { months }\end{array}$ & 100 & 72 & 22 & 6 & 94 \\
\hline $\begin{array}{c}\text { Postoperative } 6 \\
\text { months }\end{array}$ & 100 & 70 & 18 & 12 & 88 \\
\hline $\begin{array}{l}\text { Postoperative } 12 \mathrm{n} \\
\text { control group }\end{array}$ & $\begin{array}{l}\text { ths } \\
100\end{array}$ & & & & \\
\hline $\begin{array}{c}\text { Postoperative } 1 \\
\text { week }\end{array}$ & 100 & 75 & 17 & 8 & 92 \\
\hline $\begin{array}{c}\text { Postoperative } 3 \\
\text { months }\end{array}$ & 100 & 52 & 20 & 28 & 72 \\
\hline $\begin{array}{c}\text { Postoperative } 6 \\
\text { months }\end{array}$ & 100 & 42 & 24 & 34 & 66 \\
\hline $\begin{array}{c}\text { Postoperative } 12 \\
\text { months }\end{array}$ & 100 & 35 & 25 & 40 & 60 \\
\hline
\end{tabular}

\subsection{Comparison of intraocular pressure between before and after operation in two groups}

Table 2 Comparison of intraocular pressure before and after operation

\begin{tabular}{lllllll}
\hline group & \multirow{2}{*}{ preoperative } & $\begin{array}{l}\text { Postoperative } \\
\text { 1 week }\end{array}$ & $\begin{array}{l}\text { Postoperative } \\
\text { 3 months }\end{array}$ & $\begin{array}{l}\text { Postoperative } \\
6 \text { months }\end{array}$ & $\begin{array}{l}\text { Postoperative } \\
12 \text { months }\end{array}$ \\
\hline $\begin{array}{lllll}\text { observation } \\
\text { control }\end{array}$ & 100 & $37.5 \pm 2.1$ & $8.2 \pm 1.5$ & $15.2 \pm 2.5$ & $17.5 \pm 2.1$ & $18.5 \pm 2.1$ \\
\hline
\end{tabular}

\subsection{Comparison of postoperative complications between the two groups}

The incidence of postoperative complications were low in both groups. In the observation group, there were 5 cases with low intraocular pressure occurred at 2 weeks postoperatively, the incidence rate was $5 \%$, The patients with low intraocular pressure recovered gradually after one week. In the control group, there were 4 cases with low intraocular pressure occurred at 2 weeks postoperatively, the incidence rate was $4 \%$, The patients with low intraocular pressure recovered gradually after one week. there was no difference in incidence of lower intraocular pressure between the two groups. The incidence of patients with complications is low, and can be tolerated, there is no patients with corneal injury, severe complications such as malignant glaucoma, in two groups.

\subsection{Comparison of the satisfaction of treatment in two groups}

As shown in table 3, The results showed that the patients in the observation group were 
significantly more satisfied with the treatment than those in the control group.

Table 3

\begin{tabular}{|c|c|c|c|c|}
\hline group & very & satisfied & dissatisfied & total satisfied \\
\hline observation & $78 \%$ & $15 \%$ & $7 \%$ & $93 \%$ \\
\hline control & $57 \%$ & $12 \%$ & $31 \%$ & $69 \%$ \\
\hline $\mathrm{P}$ & & & & $<0.05$ \\
\hline
\end{tabular}

\section{Discussion}

This study is to compare the curative effect of trabeculectomy with mitomycin $\mathrm{C}$ and strabeculectomy alone in the treatment of glaucoma, the results show that the fully successful ratio of observation group of patients with postoperative patients is significantly higher than the control group, and the total success rate of observation group was significantly higher than that of control group, and the satisfaction rate of observation group was significantly higher than that of control group. Show that comparing the curative effect of trabeculectomy with mitomycin $\mathrm{C}$ and strabeculectomy alone in the treatment of glaucoma, trabeculectomy with mitomycin $\mathrm{C}$ has better curative effect for the treatment of glaucoma. This is consistent with previous research results. Hongmei $\mathrm{Hu}$ et al in the study, only use trabeculectomy in the control group and use trabeculectomy with mitomycin $\mathrm{C}$ in the observation group. The postoperative IOP and visual acuity were compared between the two groups. The results showed that the total effective cases of the observation group are 33, the total effective rate is as high as $94.29 \%$, the total effective cases of the control group are 22, the total effective rate was $62.86 \%$; The postoperative intraocular pressure of the control group patients within 1 year varys degrees of recovery, basic situation in the control group and 22 patients' vision of the observation group patients is improved. Visual acuity is improved in 33 cases in observation group. The comparision of effect of two groups was statistically significant $(\mathrm{P}<0.05)$. The incidence of adverse reactions in the control group was $8.57 \%$ and in the observation group was $5.71 \%$, the difference was statistically significant $(\mathrm{P}<0.05)$. Show that, based on trabeculectomy, the combination therapy of interferon and mitomycin is significant, it is worth ophthalmology clinical promotion. The results of this study are consistent with the results of this study and also show that the efficacy of trabeculectomy combined with mitomycin $\mathrm{C}$ in the treatment of glaucoma is significantly better than that of trabeculectomy alone in the treatment of glaucoma.

\section{Summary}

Trabeculectomy is a commonly used surgical treatment in the therapy of glaucoma. Although trabeculectomy is widely used in the clinical [15], but it still exists some problems. For example, postoperative trabeculectomy often due to filter passage obstruction and any other causes, lead to a failure of the patient's disease treatment, affecting the patient's visual function and quality of life. Therefore, exploring a more advanced treatment of glaucoma in the clinical is an urgent problem to be solved. Recent studies have shown that mitomycin $\mathrm{C}$ has a significant effect in the treatment of glaucoma, therefore, mitomycin $\mathrm{C}$ is currently used in the clinical treatment of glaucoma.

\section{References}

[1] Xiao-feng Liao. Therapeutic effect of trabeculectomy combined with mitomycin C on glaucoma[J]. Grassroots Medical Forum, 2016, 20(30):4301-4302.

[2] Yan Wang, Lei Ma, Du-wen Duan, et al. Efficacy of mitomycin C in combination with adjustable sutures in the treatment of glaucoma[J]. Everyone's Health: Academic Edition, 2015 (2): 141-142.

[3] Feng Pan, Jing-jing Wang, Wei Wang. Therapeutic effect of postoperative tonometry 
trabeculectomy combined with mitomycin C on glaucoma[J]. Practical anti-blind technology, 2015, 10 (2): 74-76.

[4] Na Zhang. Clinical analysis of trabeculectomy combined with mitomycin C in the treatment of glaucoma[J]. Journal of Community Medicine, 2015, 13 (19): 41-43.

[5] Quan Gu, Hong Shen. Effect of mitomycin C on intraocular pressure and ocular blood flow after glaucoma trabeculectomy [J]. International Journal of Ophthalmology, 2015 (7): 